\title{
Synthesis and characterization of an amphiphilic chitosan bearing octyl and methoxy polyethylene glycol groups
}

\author{
Guanghua Liu, Jianqun Gan, Aimin Chen, Qian Liu, Xusheng Zhao*
}

Guangzhou Institute of Chemistry, Chinese Academy of Sciences, Guangzhou, China;

*Corresponding Author: zhao7503@yahoo.com

Received 14 April 2010; revised 18 May 2010; accepted 25 May 2010.

\begin{abstract}
An amphiphilic N-octyl-O-methoxy poly (ethylene glycol) chitosan was successfully prepared by grafting successively octyl groups onto amino groups at chitosan's C-2 position as hydrophobic moieties and methoxy polyethylene glycol (MPEG) groups onto hydroxyl groups at C-6, C-3 as hydrophilic ones. A certain amount of $-\mathrm{NH}_{2}$ was retained in the structure of chitosan derivatives through protection by phthalic anhydride. The chemical structures and degree of $\mathrm{N}$-and $\mathrm{O}$-substitution of chitosan derivatives were characterized by FTIR, ${ }^{1} \mathrm{H}$ NMR, GPC and elemental analysis, respectively. The amphiphilic property for convenient self-assembly and the preserved $-\mathrm{NH}_{2}$ groups for progressive chemical cross-linking make the resultant $\mathrm{N}$ ocyl-O-MPEG chitosan soluble in water and potentially applicable in preparing stable chitosan hollow microspheres, a demanding drug-carrier in medical and pharmaceutical sciences.
\end{abstract}

Keywords: Amphiphilic Groups; Chitosan Derivatives; Protection; Hollow Microsperes

\section{INTRODUCTION}

In recent years, micelles and hollow microspheres prepared from self-assemblies of amphiphilic polymers have attracted great attention due to a variety of applications in DNA, antigens, delivery carriers for drugs and protection proteins or/and enzymes, especially for controlled or sustained drug-delivery systems [1]. The unique core-shell architecture is composed of hydrophobic segment, which acted as internal core, and hydrophilic segment which acted as surrounding corona in aqueous medium [2]. The hydrophobic core provides a loading space for water-insoluble drugs and stabilizes them, whereas the hydrophilic shell protects encapsulated drugs [3]. Additionally, the modification of hydrophilic shell affects pharmacokinetic behavior, such as prolonged circulation time, target release [4] and controlled drug release by using stimuli-sensitive copolymers [5].

In comparison with common polymers, chitosan, the second most abundant natural biopolymer only to cellulose [6] and known as its excellent property in non-toxicity, biodegradability, good biocompatibility, etc. [7], would exhibit special advantages in drug delivery system. The active hydroxyl and amino groups within chitosan could be easily modified to endow it with new or improved properties, and at the same time, keeping its original physiochemical and biochemical properties [6]. As a result, various amphiphilic chitosan derivatives and hollow microspheres prepared from them have been extensively published [6]. For examples, highly N-methylated modified chitosan possessing hydrophobic $-\mathrm{N}$ $\left(\mathrm{CH}_{3}\right)_{2},-\mathrm{NH}\left(\mathrm{CH}_{3}\right)$ and hydrophilic $-\mathrm{N}^{+}\left(\mathrm{CH}_{3}\right)_{3}$ groups was reported by Sieval et al. which is soluble in water at a broad $\mathrm{pH}$ value [8]. Through emulsion-crosslinking, Peng et al. prepared hollow microspehres from N-methylated chitosan with diameters range from 2-5 um [9]. Liu et al. synthesized an amphiphilic carboxymethylhexanoyl chitosan [10] which formed hollow nanocapsules with $20-200 \mathrm{~nm}$ in diameter [11]. As a demanding drug-carrier in medical and pharmaceutical sciences, stable chitosan hollow microsphere is probably one of the most valuable candidates.

Presently, the hydrophobic groups of amphiphilic chitosan derivatives ever reported generally contain long alkyl [12,13], long acyl [10] and aryl [14], while the hydrophilic ones include carboxymethyl [10], sulfate [12], phosphate [15,16], N-trimethyl [17] and polyethylene glycol $[14,18]$. To prepare amphiphilic chitosan 
hollow microspheres as drug carriers, the substituents, especially the hydrophilic groups, should be non-toxic and biocompatible. It's impossible to form stable corehell structure through nothing but self-assembly of amphiphilic chitosan. Amino groups within the backbone of chitosan should be crosslinked chemically to form stable and hard shell thereafter self-assembly process. That's to say, adequate primary amino groups $\left(-\mathrm{NH}_{2}\right)$ existed in amphiphilic chitosan are necessary for preparing hollow microspheres. To the best of our knowledge, most reports ever reported about amphiphilic chitosan derivatives did not pay much attention to this problem.

In this work, we report a novel amphiphilic N-octyl O-methoxy polyethylene glycol (MPEG) chitosan possessing adequate primary amino groups. PEG chain has been proved to be non-toxic, very flexible and highly hydrated, soluble in water and majority organic solvents, and is the most widely used biocompatible polymer for chemical and biological applications [19]. Through the effective protection of $-\mathrm{NH}_{2}$ groups within the backbone of chitosan during the modification process, the chitosan derivatives synthesized in this study preserve a certain amount of $-\mathrm{NH}_{2}$ groups for latter chemical crosslinking to prepare eventually sizecontrollable hollow microspheres.

\section{EXPERIMENTAL}

\subsection{Materials}

Chitosan $\left(\mathrm{Mr}=1 \times 10^{5}\right.$, degree of deacetylation $(\mathrm{DD})=$ 95\%) was provided by Zhejiang Aoxing Biochemical Co. Ltd (China). Octylaldehyde was obtained from Guangzhou Damo Chemical Co. Ltd (China). Methoxy polyethylene glycol (MPEG, Mr = 1900) was purchased from Alfa Aesar. Methyl iodide was ordered from Shanghai Jingchun Chemical Reagent Factory (China). Dimethylformamide (DMF) was distilled under reduced pressure and stored over molecular sieves. Dialysis tube was achieved from SPECTRUM (Canada) with a molecular weight cut-off (MWCO) of 6000 8000. $\mathrm{Ag}_{2} \mathrm{O}$ was freshly prepared from $\mathrm{AgNO}_{3}$ and $\mathrm{KOH}$ in our laboratory. All other materials used in this study were of analytical grade and without further purification.

\subsection{Synthetic Procedures}

\subsubsection{Synthesis of N-Octyl Chitosan (NOC)}

$\mathrm{N}$-octyl chitosan was prepared as reported previously (Scheme 1). Briefly, chitosan $(5.0 \mathrm{~g}, 31 \mathrm{mmol})$ was suspended in $60 \mathrm{ml}$ methanol with active stirring at room temperature before the addition of octaldehyde $(5.0 \mathrm{ml}$, $31 \mathrm{mmol})$. Then, $\mathrm{KBH}_{4}(2.5 \mathrm{~g})$ dissolved in $10 \mathrm{ml}$ water was added dropwise to the mixture after $24 \mathrm{~h}$. The reaction solution was neutralized with hydrochloric acid and

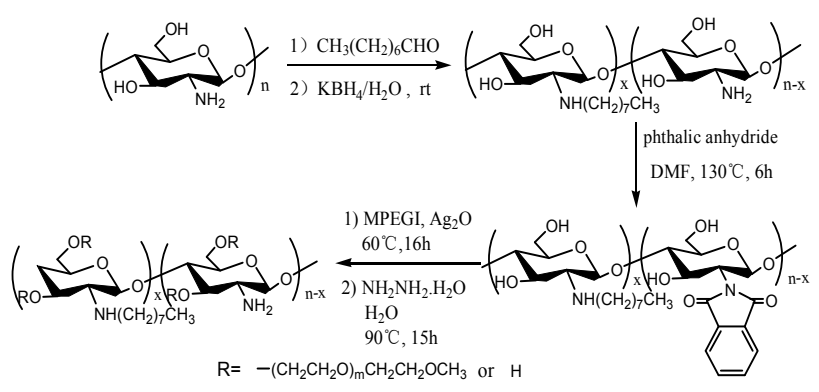

Scheme 1. Synthetic procedure of N-octyl-O-MPEG chitosan.

the product was precipitated with methanol after a further $24 \mathrm{~h}$ continuous stirring. The precipitate was filtered, washed repeatedly with methanol and water and then dried under vacuum at $60^{\circ} \mathrm{C}$ overnight to give $7.0 \mathrm{~g}$ $\mathrm{N}$-octyl chitosan. Degree of N-substitution of octyl was $54.3 \%$ from element analysis.

\subsubsection{Synthesis of N-Octyl-N-Phthaloyl Chitosan (NONPC)}

The synthesis of N-octyl-N-phthaloyl chitosan (NONPC) is described briefly as follows. A mixture of N-octyl chitosan $(3.0 \mathrm{~g}, 13.8 \mathrm{mmol})$ and phthalic anhydride (PHA) ( $2.8 \mathrm{~g}, 3$ mol equiv to amino) in $30 \mathrm{ml}$ DMF was heated to $130^{\circ} \mathrm{C}$ under nitrogen with magnetic stirring. After $6 \mathrm{~h}$, the brown precipitate obtained by pouring the solution into ice-water was collected by filtration, extracted with ethanol in Soxhlet's apparatus for $48 \mathrm{~h}$, and dried under vacuum at $50^{\circ} \mathrm{C}$ to give $3.6 \mathrm{~g}$ NONPC products.

\subsubsection{Synthesis of Methoxy Polyethylene Glycol Lodide (MPEGI)}

Methoxy polyethylene glycol iodide (MPEGI) was synthesized according to the reports by Gorochovceva and Makuska [19]. Briefly, MPEG-1900 (19 g, $10 \mathrm{mmol}$ ), triphenylphosphite $(8.0 \mathrm{ml}, 30 \mathrm{mmol})$ and methyl iodide $(4.3 \mathrm{ml}, 30 \mathrm{mmol})$ were mixed and stirred in a threeneck flask at $120^{\circ} \mathrm{C}$ for $6 \mathrm{~h}$. Then, the reaction mixture was cooled, dissolved in toluene, precipitated in $500 \mathrm{ml}$ diethyl ether, and then filtered, washed several times with diethyl ether and dried in air to give pale yellow product. The yield of the product was $92 \%$.

\subsubsection{Synthesis of N-Octyl-O-MPEG Chitosan (NOOMC)}

The synthetic procedure of N-octyl-O-MPEG chitosan (NOOMC) is carried out by using NONPC, MPEGI and $\mathrm{Ag}_{2} \mathrm{O}$ with different grams or molar ratios as the starting materials (Table 1). Firstly, NONPC, MPEGI and $\mathrm{Ag}_{2} \mathrm{O}$ were mixed and heated at $60^{\circ} \mathrm{C}$ for $16 \mathrm{~h}$ with magnetic stirring. Then, hydrazine monohydrate $(80 \%, 40 \mathrm{ml}$ or $20 \mathrm{ml})$ and distilled water $(80 \mathrm{ml}$ or $40 \mathrm{ml})$ were poured 
into the mixture and the temperature was raised to $90^{\circ} \mathrm{C}$ and maintained for $15 \mathrm{~h}$ before the mixture was filtered to remove solid impurity. The filtrate solution was dialyzed against water for $72 \mathrm{~h}$ and then concentrated before the solid residue was dried in vacuum at $50^{\circ} \mathrm{C}$ to give final products of N-octyl-O-MPEG chitosan (NOO$\mathrm{MC}$ ). Depending on different amount of hydrazine monohydrate and distilled water used during the synthesis, the products of NOOMC with two different degree of substitutions (DS) of MPEG were labeled as NOOMC (I) and NOOMC (II), respectively.

\subsection{Characterization}

${ }^{1} \mathrm{H}$ NMR spectra were recorded at $400 \mathrm{MHz}$ using a Bruker DRX-400 spectrometer. $\mathrm{CF}_{3} \mathrm{COOD}$ and $\mathrm{D}_{2} \mathrm{O}$ were used as the solvents for chitosan and its derivatives, respectively. Elemental analysis was determined using an Elementar Vario EL III analyzer. FTIR spectra were obtained from Fourier transformation infrared spectrometer (Analect RFX-65A) in $\mathrm{KBr}$ discs. Molecular weight of the amphiphilic chitosan was determined using Waters 515-410 gel permeation chromatography (GPC).

\section{RESULTS AND DISCUSSION}

\subsection{Synthesis of the Chitosan Derivatives}

Generally, amphiphilic chitosan could be prepared through introducing the hydrophilic moiety first and the hydrophobic one later $[12,13]$, or in the reverse order $[11,20]$. The latter procedure was adopted in this investigation. As shown in Scheme 1, the amphiphilic chitosan derivatives were synthesized through the following three steps. At the first step, hydrophobic octyl groups were introduced by Schiff reaction prior to MPEG. Octylaldhyde residue was found very easy to be removed with heterogeneous reaction, and rather difficult to be excluded when Schiff reaction is carried out at the last step. Meanwhile, if MPEG was introduced onto hydroxyl firstly, the molecule weight of the resultant O-MPEG chitosan would be much higher than chitosan, and the mole ratio of octylaldehyde and O-MPEG chitosan is difficult to calculate and control in Schiff reaction.

Table 1. Amounts of reactants in synthesizing NOOMC with different degree of substitutions.

\begin{tabular}{|c|c|c|c|c|c|}
\hline No. & NONPC/g & MPEGI/g & $\mathrm{Ag}_{2} \mathrm{O} / \mathrm{g}$ & $\begin{array}{l}\mathrm{m}(\mathrm{NONPC}) \\
\mathrm{m}(\mathrm{MPEGI}) \\
\mathrm{m}\left(\mathrm{Ag}_{2} \mathrm{O}\right) *\end{array}$ & product \\
\hline (I) & 1.5 & 10.2 & 1.2 & $1: 1: 1$ & $\operatorname{NOOMC}(\mathrm{I})$ \\
\hline (II) & 0.8 & 10.8 & 1.3 & $1: 2: 2$ & $\operatorname{NOOMC}(\mathrm{II})$ \\
\hline
\end{tabular}

NONPC, N-octyl-N-phthaloyl chitosan; MPEGI, methoxy polyethylene glycol iodide; NOOMC, N-octyl-O-MPEG chitosan. * m represents mole ratio.
It has been estimated that amino groups at C-2 show higher nucleophilic activity than hydroxyl groups (either C-3 or C-6) in the main backbone of chitosan [21]. Thereby, to avoid the grafting of MPEG onto amino groups which retained in N-octyl chitosan, it is necessary to protect amino groups in advance by the use of phthalic anhydride. This protection method was reported earlier in 1991 by Nishimura et al. [22], which has been applied to various region-selective modifications of chitosan [21,23]. A key point for this pathway is the fact that chitosan is insoluble in several organic solvents, such as DMF, while $\mathrm{N}$-phthaloyl derivative of chitosan is soluble [18,22]. Noctyl chitosan, just like chitosan, has also a bad solubility in organic solvent. It was found in our experiment that $\mathrm{N}$-octyl-N-phthaloyl chitosan is soluble in DMF, which is of great significance for further reaction with MPEG iodide. Accordingly, N-phthaloyl group is the prerequisite for both protection of active amino in chitosan and solubilization of the product in DMF.

As mentioned above, a certain amount of primary amino is indispensable for the preparing of amphiphilic chitosan hollow microspheres. It is identified from Scheme 1 that the content of primary amino of the final product is determined by the first step reaction. Hence, primary amino cannot be modified and replaced completely with octyl groups. In our study, to assure an incomplete substitution reaction on amino groups, we adopted an appropriate molar ratio of chitosan to octylaldehyde in Schiff reaction. It is expected that we could get amphiphilic chitosan possessing different amount of active amino groups. That is, a certain extent of primary amino content could be preserved through controlling the molar ratio of the starting materials. Further investigations are currently under way in our laboratory.

Compared with Schiff reaction at the first step, the amino groups retained in $\mathrm{N}$-octyl chitosan should be modified completely with phthalimide groups to avoid their participation in further reaction. Threefold excess of phthalic anhydride was used in our experiment to protect amino as complete as possible. The course of the etherification of N-octyl-N-phthaloyl with MPEGI was similar to that described by Natalijia Gorochovceva et al. [18] where $\mathrm{Ag}_{2} \mathrm{O}$ is a suitable and effective catalyzer for etherification. The reaction between chitosan hydroxyl groups and MPEGI is irreversible and proceeds till the consumption of active functional groups. In our study, the degree of substitution (DS) of MPEG is represented from the molar ratio of MPEGI to N-octyl-N-phthaloyl chitosan. N-octylO-MPEG chitosan with different DS of MPEG is illustrated from the molar ratio of the above two reagents.

\subsection{Characterization of Chitosan Derivatives}

The degree of substitution (DS) of chitosan derivatives was calculated by comparing $\mathrm{C}$ and $\mathrm{N}$ molar ratio ob- 
tained from the elemental analysis data (Table 1). The DS $(54.3 \%)$ of N-octyl chitosan (NOC) confirms the incomplete modification by octylaldehyde with $54.3 \%$ of amino groups substituted and about $45 \%$ of them retained in NOC. According to the variation in $\mathrm{m}(\mathrm{C}) / \mathrm{m}(\mathrm{N})$ of NOC and N-octyl-N-phthaloyl chitosan (NONPC), it can be calculated that the DS of phthlimide groups is $66.3 \%$, which exceeds the amino content (about $45 \%$ ) of NOC. This indicates that the retained amino groups were entirely protected and only a few hydroxyl groups were modified simultaneously, which is identical with the report [23] that treatment of chitosan with phthalic anhydride generally results in partial O-phthaloylation in addition to N-substitution. The DS of MPEG grafts on the final product was obtained by comparing $\mathrm{C} / \mathrm{N}$ of NOC and N-octyl-O-MPEG chitosan. As can be seen from Table 2, the DS of NOOMC (I) was only $41.8 \%$ as the molar ratio $\mathrm{m}$ (NONPC)/m(MPEGI) equals to $1: 1$. When the same molar ratio was increased up to $2: 1$, the DS of NOOMC (II) reached $88.4 \%$, more than twice as much as the DS of NOOMC (I).

Structure changes of chitosan and its derivatives were confirmed by FTIR spectra (Figure 1). The N-octylN-phthaloyl chitosan (NONPC) shows new or intensified absorptions at 2927, 2858, $1464 \mathrm{~cm}^{-1}$ which attributed to octyl chains and 1776, 1716, $721 \mathrm{~cm}^{-1}$ which assigned to the phthalimide groups. On the contrary, the peak at about $1600 \mathrm{~cm}^{-1}$, which belongs to $-\mathrm{NH}_{2}$ in chitosan, disappeared in the IR spectra of NONPC. The above information from IR spectra indicates that octyl groups were successfully introduced to chitosan and the retained amino groups were protected completely by phthalimide groups. The IR spectra (c, $d$ in Figure 1) of $\mathrm{N}$-octyl-O-MPEG chitosan (NOOMC) also reveal the absorption bands characteristic of chitosan and two modified groups. Distinctive absorption bands at $2889 \mathrm{~cm}^{-1}$ (C-H stretching) and $1110 \mathrm{~cm}^{-1}$ around belong to MPEG grafts while those at $1676,1646 \mathrm{~cm}^{-1}$ attribute to the primary amino which retained in chitosan derivative.

Table 2. Elemental analysis (\%) and the degree of substitution (DS) of chitosan derivatives.

\begin{tabular}{lccccc}
\hline \multicolumn{1}{c}{ Sample } & $\mathrm{C}$ & $\mathrm{N}$ & $\mathrm{H}$ & $\begin{array}{c}\mathrm{m}(\mathrm{C}) / \\
\mathrm{m}(\mathrm{N}))^{*}\end{array}$ & DS (\%) \\
\hline Chitosan & 39.68 & 7.59 & 7.85 & 6.10 & - \\
NOC & 53.50 & 5.98 & 9.19 & 10.44 & $54.3^{\mathrm{a}}$ \\
NONPC & 55.74 & 4.13 & 6.75 & 15.75 & $66.3^{\mathrm{b}}$ \\
NOOMC (I) & 52.01 & 1.17 & 8.89 & 51.86 & $41.8^{\mathrm{c}}$ \\
NOOMC (II) & 52.62 & 0.71 & 9.47 & 86.46 & $88.4^{\mathrm{d}}$ \\
\hline
\end{tabular}

NOC, N-octyl chitosan; NONPC, N-octyl-N-phthaloyl chitosan; NOOMC, N-octyl-O-MPEG chitosan. * m represents mole ratio. ${ }^{\text {a }}$ DS of N-octyl groups; ${ }^{b}$ DS of N-phthalimide groups; ${ }^{c, d}$ DS of O-MPEG grafts.

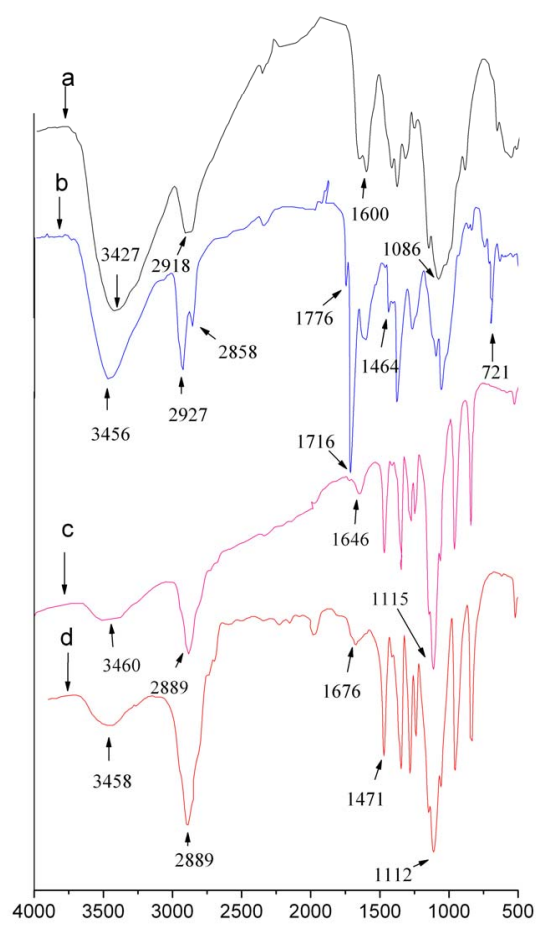

Figure 1. FTIR spectra of chitosan and its derivatives. a-chitosan, b-NONPC, c-NOOMC (I), d-NOOMC (II). NONPC, N-octyl-N-phthaloyl chitosan; NOOMC, N-octyl -O-MPEG chitosan.

It should be noted that the absorptions of octyl groups (2927, 2858, $1464 \mathrm{~cm}^{-1}$ ) were overlapped by the much stronger absorptions of MPEG grafts $\left(2889 \mathrm{~cm}^{-1}, 1471\right.$ $\left.\mathrm{cm}^{-1}\right)$. The peak at $3458 \mathrm{~cm}^{-1}$ which attributed to $-\mathrm{NH}_{2}$ and $-\mathrm{OH}$ declined and that at $1776 \mathrm{~cm}^{-1}$ and $1716 \mathrm{~cm}^{-1}$ characteristic of phthalimide groups disappeared in the FTIR spectra of the final product. It is thus concluded that MPEG groups were introduced to hydroxyl and the protecting groups were removed successfully.

More information about chitosan and its derivatives can be obtained from ${ }^{1} \mathrm{H}$ NMR analysis (Figures 2-3). In comparison with the spectra of chitosan itself (Figure 2), ${ }^{1} \mathrm{H}$ NMR spectra of NOOMC (II) (Figure 3) differ greatly in that the signals at 3.50-3.60 and 3.25 which assigned to the ethoxyl hydrogen $\left(\mathrm{CH}_{2}-\mathrm{CH}_{2}-\mathrm{O}\right)$ and methoxyl hydrogen $\left(-\mathrm{OCH}_{3}\right)$ of MPEG grafts, respectively, and that the small peaks at 3.10, 2.80, 1.15 which attributed to the methylene hydrogen around nitrogen $\left(\mathrm{N}-\mathrm{CH}_{2}-\left(\mathrm{CH}_{2}\right)_{6}-\mathrm{CH}_{3}\right)$, other six methylene hydrogens $\left(\mathrm{N}-\mathrm{CH}_{2}-\left(\mathrm{CH}_{2}\right)_{6}-\mathrm{CH}_{3}\right)$ and the methyl hydrogen $\left(\mathrm{N}-\mathrm{CH}_{2}-\right.$ $\left.\left(\mathrm{CH}_{2}\right)_{6}-\mathrm{CH}_{3}\right)$, respectively. The signals of hydrogen within chitosan backbone (3.60-3.80, H3 H4 H5 H6) were covered partially by that of ethoxyl hydrogen. As illustrated in Figure 3, the peaks of hydrogen in octyl groups and chitosan backbone were not so obvious as those of MPEG with a high DS of MPEG grafts and a 


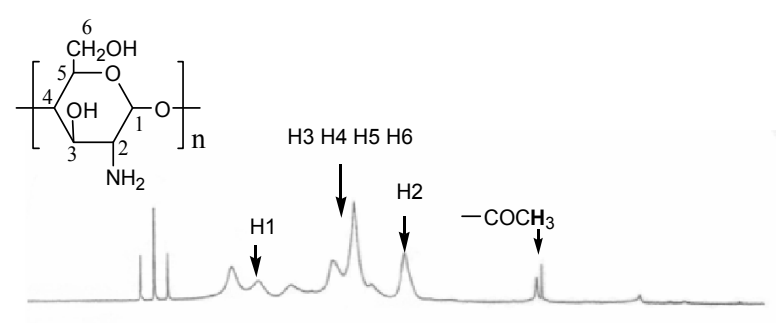

$\begin{array}{llllll}1 & 5 & 4 & 3 & 2 & 1\end{array}$

Figure 2. ${ }^{1} \mathrm{H}$ NMR spectra of chitosan.

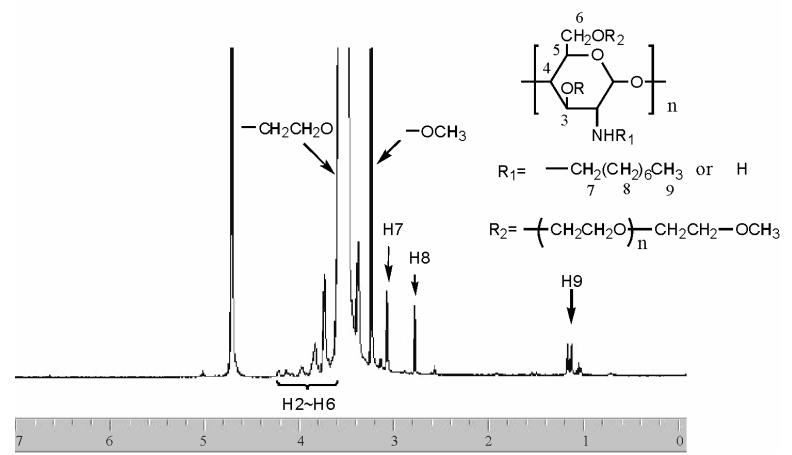

Figure 3. ${ }^{1} \mathrm{H}$ NMR spectra of N-octyl-O-MPEG chitosan (NOOMC II).

much large number of hydrogen it contained.

Molecular weight of the amphiphilic chitosan (NOOMC (I)) was measured by gel permeation chromatography (GPC). The results showed that $\mathrm{Mw}$ of NOOMC (I) is near $1 \times 10^{5}$ and Mz is about $1.6 \times 10^{5}$, which is a little bit smaller than that calculated by Element Analysis $\left(2 \times 10^{5}\right)$. It might be caused by the existence of trace amount of unreacted MPEGI after dialysis or cleavage of chitosan chains during modifications.

\section{CONCLUSIONS}

N-octyl-O-MPEG chitosan (NOOMC), was synthesized for the first time with fair production yield and high degree of substitution of amphiphilic groups. The degree of substitution of octyl groups reached $54.3 \%$ while that of MPEG grafts amounted to $41.8 \%-88.4 \%$ depending on the variation of N-octyl-N-phthaloyl chitosan to methoxy polyethylene glycol iodide molar ratio. With the introduction of the amphiphilic groups, the chitosan derivatives were endowed with good solubility in water and potential self-assembly properties. Significantly, the effective protection of amino groups was successfully adopted to retain a certain amount of $-\mathrm{NH}_{2}$ in the derivatives, which is indispensable for preparing hollow microspheres.

\section{ACKNOWLEDGEMENTS}

The authors express their gratitude to National Natural Science Foundations of China and Natural Science Foundation of Guangdong Province, China, for financial support.

\section{REFERENCES}

[1] Peng, X.H., Zhang, L.N. and Kennedy, J.F. (2006) Release behavior of microspheres from cross-linked Nmethylated chitosan encapsulated ofloxacin. Carbohydrate Polymers, 65(3), 288-295.

[2] Ye, Y.Q., Yang, F.L., Hu, F.Q., Du, Y.Z., Yuan, H. and Yu, H.Y. (2008) Core-modified chitosan-based polymeric micelles for controlled release of doxorubicin. International Journal of Pharmaceutics, 352(1-2), 294-301.

[3] van Nostrum, C.F. (2004) Polymeric micelles to deliver photosensitizers for photodynamic therapy. Advanced Drug Delivery Reviews, 56(1), 9-16.

[4] Maeda, H., Wu, J., Sawa, T., Matsumura, Y. and Hori, K. (2000) Tumor vascular permeability and the EPR effect in macromolecular therapeutics: A review. Journal of Controlled Release, 65(1-2), 271-284.

[5] Liu, X.M., Wang, L.S., Wang, L., Huang, J.C. and He, C.B. (2004) The effect of salt and $\mathrm{pH}$ on the phasetransition behaviors of temperature-sensitive copolymers based on $\mathrm{N}$-isopropylacrylamide. Biomaterials, 25(25), 5659-5666.

[6] Mourya, V.K. and Inamdar, N.N. (2008) Chitosan-modifications and applications: Opportunities galore. Reactive \& Functional Polymers, 68(6), 1013-1051.

[7] Chandy, T. and Sharma, C.P. (1990) Chitosan - as a Biomaterial. Biomaterials Artificial Cells and Artificial Organs, 18(1), 1-24.

[8] Sieval, A.B., Thanou, M., Kotze, A.F., Verhoef, J.E., Brussee, J. and Junginger, H.E. (1998) Preparation and NMR characterization of highly substituted N-trimethyl chitosan chloride. Carbohydrate Polymers, 36(2-3), 157165.

[9] Peng, X.H. and Zhang, L. (2005) Surface fabrication of hollow microspheres from N-methylated chitosan crosslinked with gultaraldehyde. Langmuir, 21(3), 10911095.

[10] Liu, T.Y., Chen, S.Y., Lin, Y.L. and Liu, D.M. (2006) Synthesis and characterization of amphiphatic carboxymethyl-hexanoyl chitosan hydrogel: Water-retention ability and drug encapsulation. Langmuir, 22(23), 97409745.

[11] Liu, K.H., Chen, S.Y., Liu, D.M. and Liu, T.Y. (2008) Self-assembled hollow nanocapsule from amphiphatic carboxymethyl-hexanoyl chitosan as drug carrier. $\mathrm{Ma}$ cromolecules, 41(17), 6511-6516.

[12] Zhang, C., Ping, Q.N., Zhang, H.J. and Jian, S. (2003) Preparation of N-alkyl-O-sulfate chitosan derivatives and micellar solubilization of taxol. Carbohydrate Polymers, 54(2), 137-141.

[13] Zhang, C., Ding, Y., Yu, L.L. and Ping, Q.N. (2007) Polymeric micelle systems of hydroxycamptothecin based on amphiphilic N-alkyl-N-trimethyl chitosan derivatives. Colloids and Surfaces B-Biointerfaces, 55(2), 192-199.

[14] Yoksan, R. and Chirachanchai, S. (2008) Amphiphilic 
chitosan nanosphere: Studies on formation, toxicity, and guest molecule incorporation. Bioorganic \& Medicinal Chemistry, 16(5), 2687-2696.

[15] Jayakumar, R., Nagahama, H., Furuike, T. and Tamura, H. (2008) Synthesis of phosphorylated chitosan by novel method and its characterization. International Journal of Biological Macromolecules, 42(4), 335-339.

[16] Jayakumar, R., Egawa, T., Furuike, T., Nair, S.V. and Tamura, H. (2009) Synthesis, characterization, and thermal properties of phosphorylated chitin for biomedical applications. Polymer Engineering and Science, 49(5), 844-849.

[17] Zhang, C., Ding, Y., Ping, Q.E. and Yu, L.L. (2006) Novel chitosan-derived nanomaterials and their micelleforming properties. Journal of Agricultural and Food Chemistry, 54(22), 8409-8416.

[18] Gorochovceva, N. and Makuska, R. (2004) Synthesis and study of water-soluble chitosan-O-poly (ethylene glycol) graft copolymers. European Polymer Journal, 40(4), 685-691.

[19] Kulbokaite, R., Ciuta, G., Netopilik, M. and Makuska, R. (2009) N-PEG'ylation of chitosan via "click chemistry" reactions. Reactive \& Functional Polymers, 69(10), 771-778.

[20] Ngawhirunpat, T., Wonglertnirant, N., Opanasopit, P., Ruktanonchai, U., Yoksan, R., Wasanasuk, K. and Chirachanchai, S. (2009) Incorporation methods for cholic acid chitosan-g-mPEG self-assembly micellar sys- tem containing camptothecin. Colloids and Surfaces B-Biointerfaces, 74(1), 253-259.

[21] Makuska, R. and Gorochovceva, N. (2006) Regioselective grafting of poly(ethylene glycol) onto chitosan through C-6 position of glucosamine units. Carbohydrate Polymers, 64(2), 319-327.

[22] Nishimura, S.I., Kohgo, O., Kurita, K. and Kuzuhara, H. (1991) Chemospecific manipulations of a rigid polysaccharide-syntheses of novel chitosan derivatives with excellent solubility in common organic-solvents by regioselective chemical modifications. Macromolecules, 24(17), 4745-4748.

[23] Hu, Y.Q., Jiang, H.L., Xu, C.N., Wang, Y.J. and Zhu, K.J. (2005) Preparation and charac terization of poly (ethylene glycol)-g-chitosan with water- and organosolubility. Carbohydrate Polymers, 61(4), 472-479. 